hep-ph/9710213 Saclay/SPhT-T97/109v2

\title{
Antenna Factorization of Gauge-Theory Amplitudes
}

\author{
David A. Kosower \\ Service de Physique Théorique \\ Centre d'Etudes de Saclay \\ F-91191 Gif-sur-Yvette cedex, France \\ kosower@spht.saclay.cea.fr
}

\begin{abstract}
I derive a single factorization formula which summarizes all soft and collinear divergences of a tree-level gauge theory amplitude. The singular factor squared is in a certain sense the generalization of the Catani-Seymour dipole factorization formula.
\end{abstract}

Submitted to Physical Review D

${ }^{\natural}$ Laboratory of the Direction des Sciences de la Matière of the Commissariat à l'Energie Atomique of France. 


\section{Introduction}

The factorization properties of gauge-theory amplitudes in infrared-singular limits have played an important role not only in our conceptual understanding of infrared safety, but also in explicit calculations at next-to-leading order in perturbative QCD. These infrared singularities may be classified into two types: soft and collinear. The former arises when a gluon four-momentum vanishes, $k_{s} \rightarrow 0$; the latter when the momenta of two massless particles become proportional, $k_{a} \rightarrow z\left(k_{a}+k_{b}\right), k_{b} \rightarrow(1-z)\left(k_{a}+k_{b}\right)$. The properties of non-Abelian gauge-theory amplitudes in these limits are easiest to understand in the context of a color decomposition [1]. For tree-level all-gluon amplitudes in an $S U(N)$ gauge theory the color decomposition has the form,

$$
\mathcal{A}_{n}^{\text {tree }}\left(\left\{k_{i}, \lambda_{i}, a_{i}\right\}\right)=\sum_{\sigma \in S_{n} / Z_{n}} \operatorname{Tr}\left(T^{a_{\sigma(1)}} \cdots T^{a_{\sigma(n)}}\right) A_{n}^{\text {tree }}\left(\sigma\left(1^{\lambda_{1}}, \ldots, n^{\lambda_{n}}\right)\right)
$$

where $S_{n} / Z_{n}$ is the group of non-cyclic permutations on $n$ symbols, and $j^{\lambda_{j}}$ denotes the $j$-th momentum and helicity. As is by now standard, I use the normalization $\operatorname{Tr}\left(T^{a} T^{b}\right)=\delta^{a b}$. One can write analogous formulæ for amplitudes with quark-antiquark pairs or uncolored external lines. The color-ordered or partial amplitude $A_{n}$ is gauge invariant, and has simple factorization properties in both the soft and collinear limits,

$$
\begin{aligned}
& A_{n}^{\text {tree }}\left(\ldots, a^{\lambda_{a}}, b^{\lambda_{b}}, \ldots\right) \stackrel{a \| b}{\longrightarrow} \sum_{\lambda= \pm} \operatorname{Split}_{-\lambda}^{\text {tree }}\left(a^{\lambda_{a}}, b^{\lambda_{b}}\right) A_{n-1}^{\text {tree }}\left(\ldots,(a+b)^{\lambda}, \ldots\right), \\
& A_{n}^{\text {tree }}\left(\ldots, a, s^{\lambda_{s}}, b, \ldots\right) \stackrel{k_{s} \rightarrow 0}{\longrightarrow} \operatorname{Soft}^{\text {tree }}\left(a, s^{\lambda_{s}}, b\right) A_{n-1}^{\text {tree }}(\ldots, a, b, \ldots) .
\end{aligned}
$$

The collinear splitting amplitude Split ${ }^{\text {tree }}$, squared and summed over helicities, gives the usual unpolarized Altarelli-Parisi splitting function [2]. While the complete amplitude also factorizes in the collinear limit, the same is not true of the soft limit; the eikonal factors Soft ${ }^{\text {tree }}$ get tangled up with the color structure. It is for this reason that the color decomposition is useful. Its use, and the simple factorization properties of the partial amplitudes, allowed Giele and Glover, in their pioneering paper [3], to derive simple and universal functions summarizing the integration over soft and collinear radiation. Of course, it is possible, as was done by later authors [4,5], to square the amplitude first, and tangle up the color factors with the eikonal factors, thereby recovering earlier forms of these two factorizations $[2,6]$, but this obscures the clean structure in eqn. (1.2).

The color decomposition (1.1) is also the form in which one writes tree-level amplitudes in an open string theory, and the gauge-theory partial amplitudes may be computed as the infinite-tension 
limit of the corresponding string amplitudes. These are given by the Koba-Nielsen formula [7],

$$
\begin{aligned}
A_{n}^{\mathrm{KN}}=\tau^{n-4} 2^{-n / 2} & \int_{x_{a}<x_{1}<x_{b} \cdots<x_{n-2}} \prod_{i=1}^{n-3} d x_{i}\left(x_{b}-x_{a}\right)\left(x_{n-2}-x_{a}\right)\left(x_{n-2}-x_{b}\right) \\
& \times\left.\prod_{i \neq j \in E}\left|x_{j}-x_{i}\right|^{\tau^{2} k_{i} \cdot k_{j} / 2} \exp \left[\sum_{i \neq j} \frac{1}{2} \frac{\varepsilon_{i} \cdot \varepsilon_{j}}{\left(x_{j}-x_{i}\right)^{2}}+\frac{\tau k_{i} \cdot \varepsilon_{j}}{\left(x_{i}-x_{j}\right)}\right]\right|_{\text {multilinear }},
\end{aligned}
$$

which I have written in a form that will be useful for the derivation to be presented in this paper. In this equation, $\left\{k_{i}, \varepsilon_{i}\right\}$ are the momenta and polarization vectors of the gluons; $E$ denotes the set of all external legs, $\{a, 1, b, 2, \ldots, n-2\} ; \tau$ is the square root of the inverse string tension; and the subscript 'multilinear' tells us to extract the terms in the exponential linear in each of the polarization vectors. Three of the $x_{i}$ may be fixed at will; it will be convenient to choose $x_{a}=0$ and $x_{b}=1$. The gauge theory limit is given by $\tau \rightarrow 0$. This formula gives an explicit form for the $n$-gluon amplitude, and examining its limits is thus a convenient method of deriving [8] factorization formulæ such as those in eqn. (1.2).

More recently, Catani and Seymour [5] wrote down a dipole factorization formula which gives a single function capturing the singular behavior of the squared matrix element in both the soft and collinear limits. Of course, since it is at the level of the amplitude squared rather than the amplitude, and since it does not take advantage of the color decomposition, it is not quite a true factorization but is still tangled up with the color algebra. The purpose of this note is to derive a single function unifying the soft and collinear factors in eqn. (1.2) at the level of color-ordered amplitudes. This will provide a true factorization. I will discuss its connection with the CataniSeymour dipole factorization formula later. The derivations in ref. [8] treat the soft and collinear limits independently.

\section{Derivation of a Unifying Factorization Formula}

Before embarking upon the derivation, I note that the Koba-Nielsen form is valid whatever the dimensions of the external momenta and polarization vectors, so long as the former are onshell, and the latter transverse. It thus allows for a treatment corresponding to any of the possible variants of dimensional regularization: the conventional scheme (CDR) [9], the original 't HooftVeltman scheme (HV) [10], dimensional reduction (DR) [11], or the four-dimensional helicity scheme $(\mathrm{FDH})[12]$.

The forms of the collinear and soft factorization functions suggest that we can associate the singular limits with a trio $(a, 1, b)$ of color-ordered partons: either a soft parton 1 with two hard 
neighbors $(a, b)$, or a pair of collinear partons neighboring another hard parton, $a \| 1$ next to $b$ or $1 \| b$ next to $a$. This in turn suggests generalizing the derivation in ref. [8] to extract all singular behavior in either of the two invariants $s_{a 1}$ or $s_{1 b}$. This will summarize the singular behavior whenever the trio of momenta become degenerate without either $a$ or $b$ becoming soft, that is as $\Delta(a, 1, b) / s_{a b}^{3} \rightarrow 0 . \quad(\Delta(a, 1, b)$ is the Gram determinant of the $(a, 1, b)$ system. $)$ In contrast, the derivation of the soft limit in ref. [8] takes both $s_{a 1}$ and $s_{1 b}$ to be small, and the derivation of the collinear limit takes only one invariant ( say $s_{a 1}$ ) to be small. The derivation below otherwise follows the same general lines: study the singular behavior of an amplitude, and identify the parts of the singular expansion that give rise to an amplitude with fewer external legs.

Let us then evaluate equation (1.3), focussing first on the singular behavior in either of the two invariants $s_{a 1}$ or $s_{1 b}$. Within this singular limit, we may also simplify expressions by expanding around the field-theory limit $\tau \rightarrow 0$. If we examine the equation, we see that singularities in these two invariants can arise only from the integration region $x_{1} \simeq x_{a}=0$ or $x_{1} \simeq x_{b}=1$, and then only in those terms which have a lone inverse power of $x_{1}$ or $1-x_{1}$ coming from the expansion of the exponential. (The integrals of $x^{-2+\delta}$ or $(1-x)^{-2+\delta}$ are finite by analytic continuation as $\delta \rightarrow 0$.) Separating out all terms containing the integration variable $x_{1}$, we obtain

$$
\begin{aligned}
\tau^{n-4} 2^{-n / 2} & \int_{x_{a}=0<x_{1}<x_{b}=1 \cdots<x_{n-2}} \prod_{i=1}^{n-3} d x_{i}\left(x_{b}-x_{a}\right)\left(x_{n-2}-x_{a}\right)\left(x_{n-2}-x_{b}\right) \\
& \times x_{1}^{\tau^{2} k_{a} \cdot k_{1}}\left(1-x_{1}\right)^{\tau^{2} k_{b} \cdot k_{1}} \prod_{j \in\{2, \ldots, n-2\}}\left(x_{j}-x_{1}\right)^{\tau^{2} k_{1} \cdot k_{j}} \\
& \times\left\{\operatorname { e x p } \left[\frac{\varepsilon_{a} \cdot \varepsilon_{1}}{x_{1}^{2}}+\frac{\varepsilon_{b} \cdot \varepsilon_{1}}{\left(1-x_{1}\right)^{2}}-\frac{\tau k_{a} \cdot \varepsilon_{1}}{x_{1}}+\frac{\tau k_{b} \cdot \varepsilon_{1}}{\left(1-x_{1}\right)}+\frac{\tau k_{1} \cdot \varepsilon_{a}}{x_{1}}-\frac{\tau k_{1} \cdot \varepsilon_{b}}{\left(1-x_{1}\right)}\right.\right. \\
& \left.\quad+\sum_{i \in\{2, \ldots, n-2\}}\left(\frac{\varepsilon_{i} \cdot \varepsilon_{1}}{\left(x_{i}-x_{1}\right)^{2}}+\frac{\tau k_{i} \cdot \varepsilon_{1}}{\left(x_{i}-x_{1}\right)}-\frac{\tau k_{1} \cdot \varepsilon_{i}}{\left(x_{i}-x_{1}\right)}\right)\right] \\
& \left.\times \prod_{i \neq j \in H}\left|x_{j}-x_{i}\right|^{\tau^{2} k_{i} \cdot k_{j} / 2} \exp \left[\sum_{i \neq j \in H} \frac{1}{2} \frac{\varepsilon_{i} \cdot \varepsilon_{j}}{\left(x_{j}-x_{i}\right)^{2}}+\frac{\tau k_{i} \cdot \varepsilon_{j}}{\left(x_{i}-x_{j}\right)}\right]\right\}\left.\right|_{\text {multilinear }}
\end{aligned}
$$

where $H=\{a, b, 2, \ldots, n-2\}$ denotes the collection of external legs excluding leg 1 . The terms on the last line form the integrand of the $(n-1)$-point amplitude, with leg 1 excluded. These will carry along with them $(n-5)$ powers of $\tau$, leaving one to be associated with the $x_{1}$ integral. Noting that we can partial-fraction terms containing inverse powers of both $x_{1}$ and $\left(1-x_{1}\right)$, we see that the $x_{1}$ integral itself can produce at most two inverse powers of $\tau$. (Each inverse power of an invariant comes along with two inverse powers of $\tau$, but the invariants cannot appear together 
in the denominator of any given term.) Thus terms in the integrand containing more than a single additional power of $\tau$ will disappear in the gauge-theory limit. This disposes of the $\prod_{j \in\{2, \ldots, n-2\}}$ factors on the second line, after expansion in powers of $x_{1}$. Using this observation in expanding the first exponential as well, in addition to dropping terms that have no inverse powers of $x_{1}$ or $\left(1-x_{1}\right)$ or are not linear in $\varepsilon_{1}$, we find,

$$
\begin{aligned}
& \tau^{n-4} 2^{-n / 2} \int_{x_{a}=0<x_{1}<x_{b}=1 \cdots<x_{n-2}} \prod_{i=1}^{n-3} d x_{i}\left(x_{b}-x_{a}\right)\left(x_{n-2}-x_{a}\right)\left(x_{n-2}-x_{b}\right) x_{1}^{\tau^{2} k_{a} \cdot k_{1}}\left(1-x_{1}\right)^{\tau^{2} k_{b} \cdot k_{1}} \\
& \times\left\{\left[\left[1-\sum_{i \in\{2, \ldots, n-2\}} \frac{\tau k_{1} \cdot \varepsilon_{i}}{\left(x_{i}-x_{1}\right)}\right]\left[\frac{\varepsilon_{a} \cdot \varepsilon_{1}}{x_{1}^{2}}+\frac{\varepsilon_{b} \cdot \varepsilon_{1}}{\left(1-x_{1}\right)^{2}}\right]\right.\right. \\
& -\frac{\tau k_{1} \cdot \varepsilon_{b}}{\left(1-x_{1}\right)} \frac{\varepsilon_{a} \cdot \varepsilon_{1}}{x_{1}^{2}}+\frac{\tau k_{1} \cdot \varepsilon_{a}}{x_{1}} \frac{\varepsilon_{b} \cdot \varepsilon_{1}}{\left(1-x_{1}\right)^{2}}-\frac{\tau k_{a} \cdot \varepsilon_{1}}{x_{1}}+\frac{\tau k_{b} \cdot \varepsilon_{1}}{\left(1-x_{1}\right)} \\
& \left.+\left[\frac{\tau k_{1} \cdot \varepsilon_{a}}{x_{1}}-\frac{\tau k_{1} \cdot \varepsilon_{b}}{\left(1-x_{1}\right)}\right] \sum_{i \in\{2, \ldots, n-2\}} \frac{\varepsilon_{i} \cdot \varepsilon_{1}}{\left(x_{i}-x_{1}\right)^{2}}\right]_{\text {s.p. }} \exp \left[-\sum_{i \in\{2, \ldots, n-2\}} \frac{\tau k_{1} \cdot \varepsilon_{i}}{\left(x_{i}-x_{1}\right)}\right] \\
& \left.\times \prod_{i \neq j \in H}\left|x_{j}-x_{i}\right|^{\tau^{2} k_{i} \cdot k_{j} / 2} \exp \left[\sum_{i \neq j \in H} \frac{1}{2} \frac{\varepsilon_{i} \cdot \varepsilon_{j}}{\left(x_{j}-x_{i}\right)^{2}}+\frac{\tau k_{i} \cdot \varepsilon_{j}}{\left(x_{i}-x_{j}\right)}\right]\right\}\left.\right|_{\text {multilinear }} .
\end{aligned}
$$

The terms with two inverse powers of $x_{1}$ or $1-x_{1}$ will not give rise to poles after the standard analytic continuation in the invariants. We can thus drop them inside the first set of brackets. This subtraction is indicated by the subscript 's.p.'. (It is equivalent to freezing $x_{1}$ at either 0 or 1 as appropriate, in the exponential on the penultimate line.)

Partial-fraction to isolate the singularities in the $x_{1}$ integral and expand the non-singular 
factors as appropriate either around $x_{1} \simeq 0$ or $x_{1} \simeq 1$,

$$
\begin{aligned}
& \tau^{n-4} 2^{-n / 2} \int_{x_{a}=0<x_{1}<x_{b}=1 \cdots<x_{n-2}} \prod_{i=1}^{n-3} d x_{i}\left(x_{b}-x_{a}\right)\left(x_{n-2}-x_{a}\right)\left(x_{n-2}-x_{b}\right) x_{1}^{\tau^{2} k_{a} \cdot k_{1}}\left(1-x_{1}\right)^{\tau^{2} k_{b} \cdot k_{1}} \\
& \times\left\{\left[\frac{1}{x_{1}^{2}}\left[1-\tau k_{1} \cdot \varepsilon_{b}-\sum_{i \in\{2, \ldots, n-2\}} \frac{\tau k_{1} \cdot \varepsilon_{i}}{x_{i}}\right] \varepsilon_{a} \cdot \varepsilon_{1}\right.\right. \\
& +\frac{1}{\left(1-x_{1}\right)^{2}}\left[1+\tau k_{1} \cdot \varepsilon_{a}-\sum_{i \in\{2, \ldots, n-2\}} \frac{\tau k_{1} \cdot \varepsilon_{i}}{\left(x_{i}-1\right)}\right] \varepsilon_{b} \cdot \varepsilon_{1} \\
& +\frac{1}{x_{1}}\left(\left[-\tau k_{1} \cdot \varepsilon_{b}-\sum_{i \in\{2, \ldots, n-2\}} \frac{\tau k_{1} \cdot \varepsilon_{i}}{x_{i}^{2}}\right] \varepsilon_{a} \cdot \varepsilon_{1}+\tau k_{1} \cdot \varepsilon_{a} \varepsilon_{b} \cdot \varepsilon_{1}-\tau k_{a} \cdot \varepsilon_{1}\right. \\
& \left.+\tau k_{1} \cdot \varepsilon_{a} \sum_{i \in\{2, \ldots, n-2\}} \frac{\varepsilon_{i} \cdot \varepsilon_{1}}{x_{i}^{2}}\right) \\
& +\frac{1}{\left(1-x_{1}\right)}\left(\left[+\tau k_{1} \cdot \varepsilon_{a}+\sum_{i \in\{2, \ldots, n-2\}} \frac{\tau k_{1} \cdot \varepsilon_{i}}{\left(x_{i}-1\right)^{2}}\right] \varepsilon_{b} \cdot \varepsilon_{1}-\tau k_{1} \cdot \varepsilon_{b} \varepsilon_{a} \cdot \varepsilon_{1}+\tau k_{b} \cdot \varepsilon_{1}\right. \\
& \left.\left.-\tau k_{1} \cdot \varepsilon_{b} \sum_{i \in\{2, \ldots, n-2\}} \frac{\varepsilon_{i} \cdot \varepsilon_{1}}{\left(x_{i}-1\right)^{2}}\right)\right] \exp \left[-\sum_{i \in\{2, \ldots, n-2\}} \frac{\tau k_{1} \cdot \varepsilon_{i}}{\left(x_{i}-x_{1}\right)}\right] \\
& \left.\times \prod_{i \neq j \in H}\left|x_{j}-x_{i}\right|^{\tau^{2} k_{i} \cdot k_{j} / 2} \exp \left[\sum_{i \neq j \in H} \frac{1}{2} \frac{\varepsilon_{i} \cdot \varepsilon_{j}}{\left(x_{j}-x_{i}\right)^{2}}+\frac{\tau k_{i} \cdot \varepsilon_{j}}{\left(x_{i}-x_{j}\right)}\right]\right\}\left.\right|_{\text {multilinear }} \text {. }
\end{aligned}
$$

It is also convenient at this stage to extract all the terms linear in $\varepsilon_{a}$ or $\varepsilon_{b}$ from the exponential 
on the last line, whence we obtain

$$
\begin{gathered}
\tau^{n-4} 2^{-n / 2} \int_{x_{a}=0<x_{1}<x_{b}=1 \ldots<x_{n-2}} \prod_{i=1}^{n-3} d x_{i}\left(x_{b}-x_{a}\right)\left(x_{n-2}-x_{a}\right)\left(x_{n-2}-x_{b}\right) \tau x_{1}^{\tau^{2} k_{a} \cdot k_{1}}\left(1-x_{1}\right)^{\tau^{2} k_{b} \cdot k_{1}} \\
\times\left\{\left[\frac { 1 } { x _ { 1 } } \left(\left[-k_{1} \cdot \varepsilon_{b}-\sum_{i \neq j \in\{2, \ldots, n-2\}} \frac{k_{1} \cdot \varepsilon_{i} \varepsilon_{b} \cdot \varepsilon_{j}}{x_{i}^{2}\left(x_{j}-1\right)^{2}}\right] \varepsilon_{a} \cdot \varepsilon_{1}+k_{1} \cdot \varepsilon_{a} \varepsilon_{b} \cdot \varepsilon_{1}-k_{a} \cdot \varepsilon_{1} \varepsilon_{a} \cdot \varepsilon_{b}\right.\right.\right. \\
-k_{a} \cdot \varepsilon_{1} \sum_{i \neq j \in\{2, \ldots, n-2\}} \frac{\varepsilon_{a} \cdot \varepsilon_{i} \varepsilon_{b} \cdot \varepsilon_{j}}{x_{i}^{2}\left(x_{j}-1\right)^{2}}+k_{1} \cdot \varepsilon_{a} \sum_{i \neq j \in\{2, \ldots, n-2\}} \frac{\varepsilon_{i} \cdot \varepsilon_{1} \varepsilon_{b} \cdot \varepsilon_{j}}{\left.x_{i}^{2}\left(x_{j}-1\right)^{2}\right)} \\
+\frac{1}{\left(1-x_{1}\right)}\left(\sum_{\left.k_{1} \cdot \varepsilon_{a}+\sum_{i \in\{2, \ldots, n-2\}} \frac{k_{1} \cdot \varepsilon_{i} \varepsilon_{a} \cdot \varepsilon_{j}}{\left(x_{i}-1\right)^{2} x_{j}^{2}}\right] \varepsilon_{b} \cdot \varepsilon_{1}-k_{1} \cdot \varepsilon_{b} \varepsilon_{a} \cdot \varepsilon_{1}+k_{b} \cdot \varepsilon_{1} \varepsilon_{a} \cdot \varepsilon_{b}}\right. \\
\left.\left.\quad+k_{b} \cdot \varepsilon_{1} \sum_{i \neq j \in\{2, \ldots, n-2\}} \frac{\varepsilon_{a} \cdot \varepsilon_{i} \varepsilon_{b} \cdot \varepsilon_{j}}{x_{i}^{2}\left(x_{j}-1\right)^{2}-k_{1} \cdot \varepsilon_{b}} \sum_{i \in\{2, \ldots, n-2\}} \frac{\varepsilon_{i} \cdot \varepsilon_{1} \varepsilon_{a} \cdot \varepsilon_{j}}{\left(x_{i}-1\right)^{2} x_{j}^{2}}\right)\right] \\
\times \exp \left[-\sum_{i \in\{2, \ldots, n-2\}}\left(\frac{\tau k_{1} \cdot \varepsilon_{i}}{\left(x_{i}-x_{1}\right)}+\frac{\tau k_{a} \cdot \varepsilon_{j}}{x_{i}}+\frac{\tau k_{b} \cdot \varepsilon_{j}}{\left(x_{i}-1\right)}\right)\right] \\
\left.\times \prod_{i \neq j \in H}\left|x_{j}-x_{i}\right|^{\tau^{2} k_{i} \cdot k_{j} / 2} \exp \left[\sum_{i \neq j \in\{2, \ldots, n-2\}} \frac{1}{2} \frac{\varepsilon_{i} \cdot \varepsilon_{j}}{\left(x_{j}-x_{i}\right)^{2}}+\frac{\tau k_{i} \cdot \varepsilon_{j}}{\left(x_{i}-x_{j}\right)}\right]\right\}\left.\right|_{\text {multilinear }}
\end{gathered}
$$

Next, introduce polarization vectors $\varepsilon_{\hat{a}}, \varepsilon_{\hat{b}}$ for two new momenta $k_{\hat{a}}, k_{\hat{b}}$, and replace two dot products in each term via the identity

$$
g^{\mu \nu}=-\sum_{\text {polarizations }} \varepsilon_{(h)}^{\mu} \varepsilon_{(h)}^{\nu *}
$$

where the sum runs over both physical and unphysical polarizations. The values of $k_{\hat{a}}, k_{\hat{b}}$ will be 
fixed later; for the moment, they are arbitrary. Collecting terms, we obtain

$$
\begin{aligned}
& \int_{x_{a}=0<x_{1}<x_{b}=1 \ldots<x_{n-2}}^{n-4} \prod_{i=1}^{n-n / 2} d x_{i}\left(x_{b}-x_{a}\right)\left(x_{n-2}-x_{a}\right)\left(x_{n-2}-x_{b}\right) \tau x_{1}^{\tau^{2} k_{a} \cdot k_{1}}\left(1-x_{1}\right)^{\tau^{2} k_{b} \cdot k_{1}} \\
& \times \sum_{\text {polarizations }(\hat{a}, \hat{b})}\left\{\left[\frac{1}{x_{1}} \varepsilon_{\hat{b}} \cdot \varepsilon_{b}\left(-\varepsilon_{a} \cdot \varepsilon_{1} k_{1} \cdot \varepsilon_{\hat{a}}+k_{1} \cdot \varepsilon_{a} \varepsilon_{\hat{a}} \cdot \varepsilon_{1}-k_{a} \cdot \varepsilon_{1} \varepsilon_{a} \cdot \varepsilon_{\hat{a}}\right)\right.\right. \\
& \times\left[\varepsilon_{\hat{a}}^{*} \cdot \varepsilon_{\hat{b}}^{*}+\sum_{i \neq j \in\{2, \ldots, n-2\}} \frac{\varepsilon_{\hat{a}}^{*} \cdot \varepsilon_{i} \varepsilon_{\hat{b}}^{*} \cdot \varepsilon_{j}}{\left.x_{i}^{2}\left(x_{j}-1\right)^{2}\right]} \exp \left[-\sum_{i \in\{2, \ldots, n-2\}}\left(\frac{\tau\left(k_{a}+k_{1}\right) \cdot \varepsilon_{i}}{x_{i}}+\frac{\tau k_{b} \cdot \varepsilon_{j}}{\left(x_{i}-1\right)}\right)\right]\right. \\
& +\frac{1}{\left(1-x_{1}\right)} \varepsilon_{\hat{a}} \cdot \varepsilon_{a}\left(\varepsilon_{b} \cdot \varepsilon_{1} k_{1} \cdot \varepsilon_{\hat{b}}-k_{1} \cdot \varepsilon_{b} \varepsilon_{\hat{b}} \cdot \varepsilon_{1}+k_{b} \cdot \varepsilon_{1} \varepsilon_{b} \cdot \varepsilon_{\hat{b}}\right) \\
& \times\left[\varepsilon_{\hat{a}}^{*} \cdot \varepsilon_{\hat{b}}^{*}+\sum_{i \neq j \in\{2, \ldots, n-2\}} \frac{\varepsilon_{\hat{a}}^{*} \cdot \varepsilon_{i} \varepsilon_{\hat{b}}^{*} \cdot \varepsilon_{j}}{x_{i}^{2}\left(x_{j}-1\right)^{2}}\right] \exp \left[-\sum_{i \in\{2, \ldots, n-2\}}\left(\frac{\tau k_{a} \cdot \varepsilon_{j}}{x_{i}}+\frac{\tau\left(k_{b}+k_{1}\right) \cdot \varepsilon_{j}}{\left(x_{i}-1\right)}\right)\right] \\
& \left.\times \prod_{i \neq j \in H}\left|x_{j}-x_{i}\right|^{\tau^{2} k_{i} \cdot k_{j} / 2} \exp \left[\sum_{i \neq j \in\{2, \ldots, n-2\}} \frac{1}{2} \frac{\varepsilon_{i} \cdot \varepsilon_{j}}{\left(x_{j}-x_{i}\right)^{2}}+\frac{\tau k_{i} \cdot \varepsilon_{j}}{\left(x_{i}-x_{j}\right)}\right]\right\}\left.\right|_{\text {multilinear }}
\end{aligned}
$$

where 'multilinear' is now understood to refer to $\varepsilon$ and $\varepsilon^{*}$ independently, and where I have set $x_{1}$ in the exponential to 0 or 1 as appropriate to the pole structure of each term.

Take $x_{\hat{a}}=0, x_{\hat{b}}=1$, and define $\widehat{H}=\{-\hat{a},-\hat{b}, 2, \ldots, n-2\} ;$ ' $-\hat{a}$ ' signifies the replacement of $\left(k_{\hat{a}}, \varepsilon_{\hat{a}}^{*}\right)$ by $\left(k_{-\hat{a}}, \varepsilon_{-\hat{a}}\right) \equiv\left(-k_{\hat{a}}, \varepsilon_{\hat{a}}^{*}\right)$. We can absorb the factors in brackets into the exponential on 
the last line,

$$
\begin{aligned}
& \tau^{n-4} 2^{-n / 2} \int_{x_{a}=0<x_{1}<x_{b}=1 \cdots<x_{n-2}} \prod_{i=1}^{n-3} d x_{i}\left(x_{b}-x_{a}\right)\left(x_{n-2}-x_{a}\right)\left(x_{n-2}-x_{b}\right) \tau x_{1}^{\tau^{2} k_{a} \cdot k_{1}}\left(1-x_{1}\right)^{\tau^{2} k_{b} \cdot k_{1}} \\
& \times \sum_{\text {polarizations }(\hat{a}, \hat{b})}\left\{\left[\frac{1}{x_{1}} \varepsilon_{\hat{b}} \cdot \varepsilon_{b}\left(-\varepsilon_{a} \cdot \varepsilon_{1} k_{1} \cdot \varepsilon_{\hat{a}}+k_{1} \cdot \varepsilon_{a} \varepsilon_{\hat{a}} \cdot \varepsilon_{1}-k_{a} \cdot \varepsilon_{1} \varepsilon_{a} \cdot \varepsilon_{\hat{a}}\right)\right.\right. \\
& \times \exp \left[-\sum_{i \in\{2, \ldots, n-2\}}\left(\frac{\tau\left(k_{a}+k_{1}\right) \cdot \varepsilon_{i}}{x_{i}}+\frac{\tau k_{b} \cdot \varepsilon_{j}}{\left(x_{i}-1\right)}+\frac{\tau k_{\hat{a}} \cdot \varepsilon_{i}}{x_{i}}+\frac{\tau k_{\hat{b}} \cdot \varepsilon_{j}}{\left(x_{i}-1\right)}\right)\right] \\
& +\frac{1}{\left(1-x_{1}\right)} \varepsilon_{\hat{a}} \cdot \varepsilon_{a}\left(\varepsilon_{b} \cdot \varepsilon_{1} k_{1} \cdot \varepsilon_{\hat{b}}-k_{1} \cdot \varepsilon_{b} \varepsilon_{\hat{b}} \cdot \varepsilon_{1}+k_{b} \cdot \varepsilon_{1} \varepsilon_{b} \cdot \varepsilon_{\hat{b}}\right) \\
& \left.\times \exp \left[-\sum_{i \in\{2, \ldots, n-2\}}\left(\frac{\tau k_{a} \cdot \varepsilon_{j}}{x_{i}}+\frac{\tau\left(k_{b}+k_{1}\right) \cdot \varepsilon_{j}}{\left(x_{i}-1\right)}+\frac{\tau k_{\hat{a}} \cdot \varepsilon_{j}}{x_{i}}+\frac{\tau k_{\hat{b}} \cdot \varepsilon_{j}}{\left(x_{i}-1\right)}\right)\right]\right] \\
& \left.\times \prod_{i \neq j \in H}\left|x_{j}-x_{i}\right|^{\tau^{2} k_{i} \cdot k_{j} / 2} \exp \left[\sum_{i \neq j \in \widehat{H}} \frac{1}{2} \frac{\varepsilon_{i} \cdot \varepsilon_{j}}{\left(x_{j}-x_{i}\right)^{2}}+\frac{\tau k_{i} \cdot \varepsilon_{j}}{\left(x_{i}-x_{j}\right)}\right]\right\}\left.\right|_{\text {multilinear }},
\end{aligned}
$$

up to terms which vanish in the gauge-theory limit.

If we now choose $k_{\hat{a}}=-k_{a}-k_{1}$ and $k_{\hat{b}}=-k_{b}$ in the first term, and $k_{\hat{a}}=-k_{a}$ and $k_{\hat{b}}=-k_{b}-k_{1}$ in the second, we can perform the $x_{2} \ldots x_{n-3}$ integrations. They yield an $(n-1)$-point amplitude, so that the singular limit of the original amplitude becomes

$$
\begin{aligned}
\frac{\tau^{2}}{\sqrt{2}} \int_{0}^{1} d x_{1} x_{1}^{\tau^{2} k_{a} \cdot k_{1}}\left(1-x_{1}\right)^{\tau^{2} k_{b} \cdot k_{1}} \\
\quad \times \sum_{\substack{\text { polarizations }(\hat{a}, \hat{b}) \\
x_{1}}} \frac{1}{x_{\hat{b}} \cdot \varepsilon_{b}\left(-\varepsilon_{a} \cdot \varepsilon_{1} k_{1} \cdot \varepsilon_{\hat{a}}+k_{1} \cdot \varepsilon_{a} \varepsilon_{\hat{a}} \cdot \varepsilon_{1}-k_{a} \cdot \varepsilon_{1} \varepsilon_{a} \cdot \varepsilon_{\hat{a}}\right)} \\
\quad \times A_{n-1}^{\mathrm{KN}}\left(\ldots,-\hat{a}=a+k_{1},-\hat{b}=b, \ldots\right) \\
+\frac{1}{\left(1-x_{1}\right)} \varepsilon_{\hat{a}} \cdot \varepsilon_{a}\left(\varepsilon_{b} \cdot \varepsilon_{1} k_{1} \cdot \varepsilon_{\hat{b}}-k_{1} \cdot \varepsilon_{b} \varepsilon_{\hat{b}} \cdot \varepsilon_{1}+k_{b} \cdot \varepsilon_{1} \varepsilon_{b} \cdot \varepsilon_{\hat{b}}\right) \\
\left.\quad \times A_{n-1}^{\mathrm{KN}}\left(\ldots,-\hat{a}=a,-\hat{b}=b+k_{1}, \ldots\right)\right]
\end{aligned}
$$

Performing the $x_{1}$ integration and taking the $\tau \rightarrow 0$ limit then gives us

$$
\begin{aligned}
& \sum_{\text {polarizations }(\hat{a}, \hat{b})}\left[\frac{1}{s_{a 1}} \varepsilon_{\hat{b}} \cdot \varepsilon_{b} V_{3}(a, 1, \hat{a}) A_{n-1}^{\text {tree }}\left(\ldots,-\hat{a}=a+k_{1},-\hat{b}=b, \ldots\right)\right. \\
& \left.\quad-\frac{1}{s_{b 1}} \varepsilon_{\hat{a}} \cdot \varepsilon_{a} V_{3}(b, 1, \hat{b}) A_{n-1}^{\text {tree }}\left(\ldots,-\hat{a}=a,-\hat{b}=b+k_{1}, \ldots\right)\right]
\end{aligned}
$$


where $V_{3}(a, b, c)$ is a certain form of the color-ordered three-point vertex (up to a factor of $i$ ),

$$
V_{3}(1,2,3)=\sqrt{2}\left(-\varepsilon_{1} \cdot \varepsilon_{2} k_{2} \cdot \varepsilon_{3}+k_{2} \cdot \varepsilon_{1} \varepsilon_{3} \cdot \varepsilon_{2}-k_{1} \cdot \varepsilon_{2} \varepsilon_{1} \cdot \varepsilon_{3}\right)
$$

In the singular limit, one might write down other forms of the three-point vertex. However, later formulæ will not conserve momentum amongst the three momentum in these vertices (they will of course conserve momentum overall), and as we will be taking the ratio of two quantities which both vanish in the singular limit, we must be careful which form we use. (With this in mind, one could also use the recurrence relations formalism [13] to derive eqn. (2.9).)

If we can find a pair of reconstruction functions $k_{\hat{a}, \hat{b}}=f_{\hat{a}, \hat{b}}\left(k_{a}, k_{1}, k_{b}\right)$ such that $\left(k_{\hat{a}}, k_{\hat{b}}\right) \rightarrow$ $-\left(k_{a}+k_{1}, k_{b}\right)$ sufficiently quickly when $k_{1} \| k_{a}$, and likewise $\left(k_{\hat{a}}, k_{\hat{b}}\right) \rightarrow-\left(k_{a}, k_{b}+k_{1}\right)$ sufficiently quickly when $k_{1} \| k_{b}$, then we can pull out a common factor of the surviving hard amplitude. Furthermore, gauge invariance allows us to replace the sum over polarizations with a sum over helicities, so we obtain for the singular limit,

$$
\sum_{\text {helicities }(\hat{a}, \hat{b})}\left[\frac{1}{s_{a 1}} \varepsilon_{\hat{b}} \cdot \varepsilon_{b} V_{3}(a, 1, \hat{a})-\frac{1}{s_{b 1}} \varepsilon_{\hat{a}} \cdot \varepsilon_{a} V_{3}(b, 1, \hat{b})\right] A_{n-1}^{\text {tree }}(\ldots,-\hat{a}(a, 1, b),-\hat{b}(b, 1, a), \ldots) .
$$

(In the four-dimensional helicity scheme, only the \pm helicities are needed; in the conventional and 't Hooft-Veltman schemes, ' $\epsilon$ ' helicities are needed as well.) The factor in brackets is the antenna factorization function,

$$
\operatorname{Ant}(\hat{a}, \hat{b} \longleftarrow a, 1, b)=\frac{1}{s_{a 1}} \varepsilon_{\hat{b}} \cdot \varepsilon_{b} V_{3}(a, 1, \hat{a})-\frac{1}{s_{b 1}} \varepsilon_{\hat{a}} \cdot \varepsilon_{a} V_{3}(b, 1, \hat{b})
$$

This function factorizes the singular region of three-particle production onto the antenna formed by two hard particles. It depends on the helicities of the original particles, as well as the helicities of the resulting hard particles.

\section{Explicit Forms}

In the $k_{a} \| k_{1}$ collinear limit, the second term in eqn. (2.12) is non-singular, and we recover the usual collinear splitting amplitude, given by the first term [8] (note that the normalization conventions there are slightly different from those in the present paper). Similarly, in the $k_{b} \| k_{1}$ limit, only the second term contributes. In the soft limit $\left(k_{1} \rightarrow 0\right), k_{\hat{a}}=-k_{a}, k_{\hat{b}}=-k_{b}$, and we can neglect all terms proportional to $k_{1}$ in $V_{3}$; we then obtain

$$
\varepsilon_{\hat{a}} \cdot \varepsilon_{a} \varepsilon_{b} \cdot \varepsilon_{\hat{b}}\left[-\frac{1}{s_{a 1}} k_{a} \cdot \varepsilon_{1}+\frac{1}{s_{b 1}} k_{b} \cdot \varepsilon_{1}\right]
$$


which is just the usual eikonal factor. Thus we recover known factorization formulæ in the appropriate subregions.

Sensible reconstruction functions satisfy momentum conservation everywhere, and also keep $k_{\hat{a}, \hat{b}}$ massless everywhere (not just in the singular limit). This corresponds to taking the sum of the original momenta, $K=k_{a}+k_{1}+k_{b}$, and rewriting it as the sum of two massless momenta. Working in the frame where $\mathbf{K}=0$, we see that there is a degeneracy corresponding to the relative angle of $\mathbf{b}$ and $-\hat{\mathbf{b}}$. In the singular limit, this degeneracy is fixed by the requirement that the angle vanish; but away from the limit, it survives. This means that the choice of reconstruction function is not unique.

One possibility is to use the Catani-Seymour choice,

$$
k_{\hat{a}}=-k_{a}-k_{1}+\frac{s_{a 1}}{s_{1 b}+s_{a b}} k_{b}, \quad k_{\hat{b}}=-\frac{K^{2}}{s_{1 b}+s_{a b}} k_{b},
$$

which however is asymmetric in our case (and is not valid for $k_{b} \| k_{1}$ ). A more symmetric choice is to take

$$
\begin{aligned}
& s_{\hat{a} a}=-\frac{s_{a 1} s_{1 b}}{s_{1 b}+s_{a b}} r_{1}\left[1-\frac{2\left(1-r_{1}\right)}{\rho+1}\right], \\
& s_{\hat{b} b}=-\frac{s_{a 1} s_{1 b}}{s_{1 a}+s_{a b}}\left(1-r_{1}\right)\left[1-\frac{2 r_{1}}{\rho+1}\right],
\end{aligned}
$$

where

$$
r_{1}=\frac{s_{1 b}}{s_{1 a}+s_{1 b}}, \quad \rho=\sqrt{1+\frac{4 r_{1}\left(1-r_{1}\right) s_{1 a} s_{1 b}}{K^{2} s_{a b}}} .
$$

(Note that $\rho \rightarrow 1$ in all singular limits.) This is equivalent to

$$
\begin{aligned}
& k_{\hat{a}}=-\frac{1}{2}\left[1+\rho+\frac{s_{1 b}\left(1+\rho-2 r_{1}\right)}{s_{1 a}+s_{a b}}\right] k_{a}-r_{1} k_{1}-\frac{1}{2}\left[1-\rho+\frac{s_{1 a}\left(1-\rho-2 r_{1}\right)}{s_{1 b}+s_{a b}}\right] k_{b}, \\
& k_{\hat{b}}=-\frac{1}{2}\left[1-\rho-\frac{s_{1 b}\left(1+\rho-2 r_{1}\right)}{s_{1 a}+s_{a b}}\right] k_{a}-\left(1-r_{1}\right) k_{1}-\frac{1}{2}\left[1+\rho-\frac{s_{1 a}\left(1-\rho-2 r_{1}\right)}{s_{1 b}+s_{a b}}\right] k_{b},
\end{aligned}
$$

These approach the appropriate Catani-Seymour choice in both collinear limits, $a \| 1$ and $b \| 1$.

So long as we stay in four dimensions, we can use the spinor helicity method to give a concrete expression for the antenna function. Given values for the momenta $k_{i}$, these quantities can be evaluated numerically. For example,

$$
\operatorname{Ant}\left(\hat{a}^{-}, \hat{b}^{-} \longleftarrow a^{+}, 1^{+}, b^{+}\right)=-\frac{K^{2}}{\langle a 1\rangle\langle 1 b\rangle} \frac{[a b]\langle b \hat{a}\rangle\langle a \hat{b}\rangle}{\langle a b\rangle^{2}[b \hat{a}][a \hat{b}]}
$$

where I have used identities such as

$$
\frac{\langle q \hat{a}\rangle}{\langle q a\rangle}=\frac{\langle b \hat{a}\rangle}{\langle b a\rangle}+\frac{\langle q b\rangle\langle\hat{a} a\rangle}{\langle q a\rangle\langle b a\rangle}
$$


and have dropped terms such as the second which are non-singular in the limit.

Squaring the antenna function, summing over final helicities $(a, 1, b)$, and averaging over initial helicities $(\hat{a}, \hat{b})$, one obtains

$$
2 \frac{\left[K^{2}\left(K^{2}-s_{a b}\right)+s_{a b}^{2}\right]^{2}}{s_{a 1} s_{1 b} s_{a b}\left(K^{2}\right)^{2}}+\cdots,
$$

where the omitted terms are less singular, and in particular lead to finite integrals (as $\epsilon \rightarrow 0$ ) when integrated over 'singular' (soft or collinear) regions of phase space. (The inclusion or exclusion of such terms is arbitrary, as they are not universal. In practical applications one may choose to omit them.) The reader may verify that this expression reproduces known expressions in the soft and collinear limits.

Away from four dimensions, we must take the unobserved momenta $(a, 1, b)$ to have $4-2 \epsilon$ rather than four components; depending on the variant of dimensional regularization, we may also take polarization vectors or observed momenta $(\hat{a}, \hat{b})$ to have $4-2 \epsilon$ rather than four components. In the CDR scheme, one obtains the same expression (3.8) for the antenna function; in other schemes, there will in general be additional contributions.

If instead we choose the Catani-Seymour reconstruction function, we cannot expect to reproduce the correct behavior in the $k_{b} \| k_{1}$ limit, but we will reproduce the Catani-Seymour dipole factorization in both the $k_{a} \| k_{1}$ and $k_{1} \rightarrow 0$ limits. (Although the antenna function is not equal to the Catani-Seymour dipole function, the difference between them consists of terms which are not singular in the limit. Such terms, as noted above, are not universal, and may be omitted at will.) The corresponding helicity amplitudes could be thought of as the 'square root' of the dipole function. The antenna function may be thought of as a generalization of the dipole factorization formula.

Although I have discussed only the antenna factorization function for purely gluonic amplitudes, it is clear that one can write down analogous formulae for mixed quark-gluon amplitudes. The approach presented here has a straightforward generalization to multiple-singular emission, which I shall endeavor to discuss elsewhere. I thank Z. Bern, S. Catani, L. Dixon, and N. Glover for helpful comments. 


\section{References}

[1] F. A. Berends and W. T. Giele, Nucl. Phys. B294:700 (1987);

D. A. Kosower, B.-H. Lee and V. P. Nair, Phys. Lett. 201B:85 (1988);

M. Mangano, S. Parke and Z. Xu, Nucl. Phys. B298:653 (1988);

Z. Bern and D. A. Kosower, Nucl. Phys. B362:389 (1991).

[2] G. Altarelli and G. Parisi, Nucl. Phys. B126:298 (1977).

[3] W.T. Giele and E.W.N. Glover, Phys. Rev. D46:1980 (1992).

[4] S. Frixione, Z. Kunszt, and A. Signer, Nucl. Phys. B467:399 (1996) hep-ph/9512328.

[5] S. Catani and M. Seymour, Phys. Lett. B378:287 (1996) hep-ph/9602277; Nucl. Phys. B485:291 (1997) hep-ph/9605323.

[6] A. Bassetto, M. Ciafaloni, and G. Marchesini, Phys. Rep. 100:201 (1983).

[7] Z. Koba and H. B. Nielsen, Nucl. Phys. B10:633 (1969);

J. H. Schwarz, Phys. Rep. 89:223 (1982).

[8] M. Mangano and S.J. Parke, Phys. Rep. 200:301 (1991).

[9] J. C. Collins, Renormalization (Cambridge University Press, 1984).

[10] G. 't Hooft and M. Veltman, Nucl. Phys. B44:189 (1972).

[11] W. Siegel, Phys. Lett. 84B:193 (1979);

D.M. Capper, D.R.T. Jones and P. van Nieuwenhuizen, Nucl. Phys. B167:479 (1980);

L.V. Avdeev and A.A. Vladimirov, Nucl. Phys. B219:262 (1983).

[12] Z. Bern and D. A. Kosower, Nucl. Phys. B379:451 (1992).

[13] F. A. Berends and W. T. Giele, Nucl. Phys. B306:759 (1988);

D. A. Kosower, Nucl. Phys. B335:23 (1990). 